See discussions, stats, and author profiles for this publication at: https://www.researchgate.net/publication/326032503

WHO/ILO work-related burden of disease and injury: Protocol for systematic reviews of occupational exposure to dusts and/or fibres and of the effect of occupational exposure to dus...

Article in Environment International · June 2018

DOI: 10.1016/j.envint.2018.06.005

CITATIONS

5

21 authors, including:

Daniele Mandrioli

L'Istituto Ramazzini

41 PUBLICATIONS 354 CITATIONS

SEE PROFILE

Robert A Cohen

University of Illinois at Chicago

40 PUBLICATIONS 275 CITATIONS

SEE PROFILE
123

Vivi Schlünssen

National Research Centre for the Working Environment / Aarhus University 280 PUBLICATIONS 2,497 CITATIONS

SEE PROFILE

Claudio Colosio

University of Milano \& Azienda Ospedaliera San Paolo - Polo Universitario

175 PUBLICATIONS 2,281 CITATIONS

SEE PROFILE

Some of the authors of this publication are also working on these related projects:

Dermal uptake of PAH View project

Development and validation of questionnaires for the re-integration of employees View project 
Review article

\title{
WHO/ILO work-related burden of disease and injury: Protocol for systematic reviews of occupational exposure to dusts and/or fibres and of the effect of occupational exposure to dusts and/or fibres on pneumoconiosis ${ }^{\text {th }}$
}

\author{
Daniele Mandrioli $^{\mathrm{a}, 1}$, Vivi Schlünssen ${ }^{\mathrm{b}, \mathrm{c}, 1}$, Balázs Ádám ${ }^{\mathrm{d}}$, Robert A. Cohen ${ }^{\mathrm{e}}$, Claudio Colosiof,g, \\ Weihong Chen $^{\mathrm{h}}$, Axel Fischer ${ }^{\mathrm{i}}$, Lode Godderis ${ }^{\mathrm{j}}$, Thomas Göen ${ }^{\mathrm{k}}$, Ivan D. Ivanov ${ }^{1}$, Nancy Leppink ${ }^{\mathrm{m}}$, \\ Stefan Mandic-Rajcevic ${ }^{\mathrm{f}}$, Federica Masci ${ }^{\mathrm{f}}$, Ben Nemery ${ }^{\mathrm{j}}$, Frank Pegal, Annette Prüss-Üsstün, \\ Daria Sgargi $^{\mathrm{b}}$, Yuka Ujita ${ }^{\mathrm{m}}$, Stevie van der Mierden ${ }^{\mathrm{a}}$, Muzimkhulu Zungu ${ }^{\mathrm{n}}$, \\ Paul T.J. Scheepers ${ }^{0, *, 1}$
}

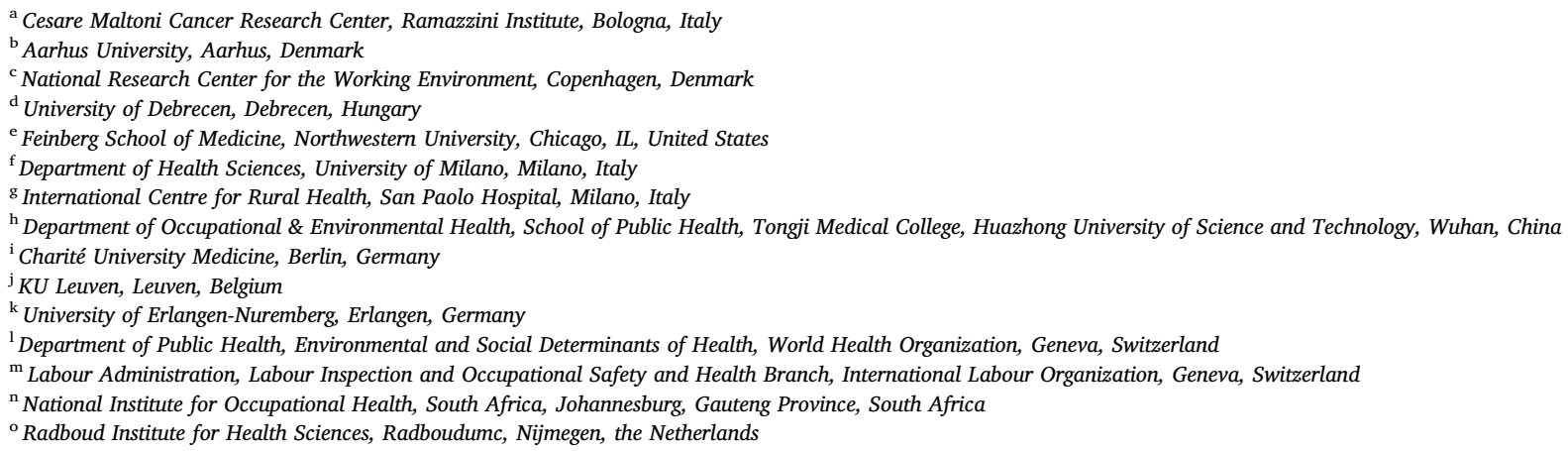

\section{A R T I C L E I N F O}

\section{Keywords:}

Asbestos

Asbestosis

Coal dust

Occupational health

Silica

Silicosis

\begin{abstract}
A B S T R A C T
Background: The World Health Organization (WHO) and the International Labour Organization (ILO) are developing a joint methodology for estimating the national and global work-related burden of disease and injury (WHO/ILO joint methodology), with contributions from a large network of experts. In this paper, we present the protocol for two systematic reviews of parameters for estimating the number of deaths and disability-adjusted life years attributable to pneumoconiosis from occupational exposure to dusts and/or fibres, to inform the development of the WHO/ILO joint methodology.

Objectives: We aim to systematically review studies on occupational exposure to dusts and/or fibres (Systematic Review 1) and systematically review and meta-analyse estimates of the effect of occupational exposure to dusts and/or fibres on pneumoconiosis (Systematic Review 2), applying the Navigation Guide systematic review methodology as an organizing framework.

Data sources: Separately for Systematic Reviews 1 and 2, we will search electronic academic databases for potentially relevant records from published and unpublished studies, including Medline, EMBASE, Web of Science and CISDOC. We will also search electronic grey literature databases, Internet search engines and organizational
\end{abstract}

\footnotetext{
Systematic review registration number: CRD42018084131.

* Corresponding author at: Radboud Institute for Health Sciences, Radboudumc, PO Box 9101, 6500 HB Nijmegen, the Netherlands.

E-mail addresses: mandriolid@ramazzini.it (D. Mandrioli), vs@ph.au.dk (V. Schlünssen), adam.balazs@sph.unideb.hu (B. Ádám), bobcohen@uic.edu (R.A. Cohen), Claudio.colosio@unimi.it (C. Colosio),wchen@mails.tjmu.edu.cn (W. Chen), axel.fischer@charite.de (A. Fischer), lode.godderis@med.kuleuven.be (L. Godderis), thomas.goeen@fau.de (T. Göen), ivanovi@who.int (I.D. Ivanov), leppink@ilo.org (N. Leppink),

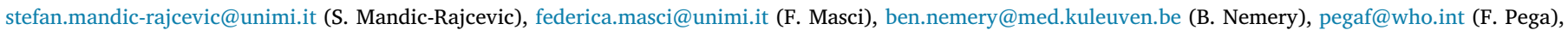
pruessa@who.int (A. Prüss-Üstün), ujita@ilo.org (Y. Ujita), Muzimkhulu.zungu@nioh.nhls.ac.za (M. Zungu), paul.scheepers@radboudumc.nl (P.T.J. Scheepers).

${ }^{1}$ Contributed equally.
} 
websites; hand-search reference list of previous systematic reviews and included study records; and consult additional experts.

Study eligibility and criteria: We will include working-age ( $\geq 15$ years) study participants in the formal and informal economy in any WHO and/or ILO Member State but exclude children ( $<15$ years) and unpaid domestic workers. Eligible risk factors will be dusts and/or fibres from: (i) asbestos; (ii) silica; and/or (iii) coal (defined as pure coal dust and/or dust from coal mining). Included outcomes will be (i) asbestosis; (ii) silicosis; (iii) coal worker pneumoconiosis; and (iv) unspecified pneumoconiosis. For Systematic Review 1, we will include quantitative prevalence studies of occupational exposure to dusts and/or fibres (i.e. no versus any exposure) stratified by country, sex, age and industrial sector or occupation. For Systematic Review 2, we will include randomized controlled trials, cohort studies, case-control studies and other non-randomized intervention studies with an estimate of any occupational exposure to dusts and/or fibres on the prevalence of, incidence of or mortality due to pneumoconiosis, compared with the theoretical minimum risk exposure level of no exposure. Study appraisal and synthesis methods: At least two review authors will independently screen titles and abstracts against the eligibility criteria at a first stage and full texts of potentially eligible records at a second stage, followed by extraction of data from qualifying studies. At least two review authors will assess risk of bias and the quality of evidence, using the most suited tools currently available. For Systematic Review 2, if feasible, we will combine relative risks using meta-analysis. We will report results using the guidelines for accurate and transparent health estimates reporting (GATHER) for Systematic Review 1 and the preferred reporting items for systematic reviews and meta-analyses guidelines (PRISMA) for Systematic Review 2.

PROSPERO registration number: CRD42018084131.

\section{Background}

The World Health Organization (WHO) and the International Labour Organization (ILO) are developing a joint methodology for estimating the work-related burden of disease and injury (WHO/ILO joint methodology) (Ryder, 2017). The organizations plan to estimate the numbers of deaths and disability-adjusted life years (DALYs) that are attributable to selected occupational risk factors. The WHO/ILO joint methodology will be based on already existing WHO and ILO methodologies for estimating the burden of disease for selected occupational risk factors (Pruss-Ustun et al., 2017; International Labour Organization, 2014). It will expand existing methodologies with estimation of the burden of several prioritized additional pairs of occupational risk factors and health outcomes. For this purpose, populationattributable fractions (Murray et al., 2004) - the proportional reduction in burden from the health outcome achieved by a reduction of exposure to the theoretical minimum risk exposure level - will be calculated for each additional risk factor-outcome pair, and these fractions will be applied to the total disease burden envelopes for the health outcome from the WHO Global Health Estimates (World Health Organization, 2017).

The WHO/ILO joint methodology may include a methodology for estimating the burden of pneumoconiosis from occupational exposure to dusts and/or fibres, if feasible, as one additional prioritized risk factor-outcome pair. To optimize parameters used in estimation models, a systematic review is required of studies on the prevalence of occupational exposure to dusts and/or fibres ('Systematic Review 1'), as well as a second systematic review and meta-analysis of studies with estimates of the effect of occupational exposure to dusts and/or fibres on pneumoconiosis ('Systematic Review 2'). In the current paper, we present the protocol for these two systematic reviews, in parallel to systematic review protocols on other additional risk factor-outcome pairs (Hulshof et al., 2018; Tenkate et al., 2018; John et al., 2018; Teixeira et al., 2018; Li et al., 2018; Descatha et al., 2018; Rugulies et al., 2018; Pachito et al., 2018). To our knowledge, this is the first protocol of its kind. The WHO/ILO joint estimation methodology and the burden of disease estimates are separate from these systematic reviews, and they will be described and reported elsewhere.

We refer separately to Systematic Reviews 1 and 2 because the two systematic reviews address different objectives and therefore require different methodologies. The two systematic reviews will, however, be harmonized and conducted in tandem. This will ensure that - in the later development of the methodology for estimating the burden of disease from this risk factor-outcome pair - the parameters on the risk factor prevalence are optimally matched with the parameters from studies on the effect of the risk factor on the designated outcome. The findings from Systematic Reviews 1 and 2 will be reported in two distinct journal articles.

\subsection{Rationale}

Exposures to asbestos, silica and coal dust (defined as pure coal dust and/or dust from coal mining) are known occupational risk factors for pneumoconiosis. In the Institute for Health Metrics and Evaluation Global Burden of Disease Study 2016, asbestosis (as an outcome separate to coal worker pneumoconiosis and other pneumoconiosis) and silicosis are $100 \%$ attributed to occupational exposure to asbestos and silica respectively (G. B. D. Risk Factors Collaborators, 2017). In the same study, the entire burden of coal worker pneumoconiosis and of other pneumoconiosis is $100 \%$ attributed to the risk factor occupational particulate matter, gases and fumes (G. B. D. Risk Factors Collaborators, 2017). However, the population-attributable fractions may actually be smaller than 1.00 , considering that some burden of pneumoconiosis may be caused by residential exposure to one or more sources of asbestos (Tarres et al., 2013), silica and coal dust (Akaoka et al., 2017) among residents near mines; non-occupational exposure to silica from the natural environment (e.g. in deserts or from sand storms) (De Berardis et al., 2007) and from second-hand exposures (e.g. family members of exposed workers coming into contact with contaminated clothes etc.).

To consider the feasibility of estimating the burden of pneumoconiosis from occupational exposure by inhalation of dusts and/or fibres, and to ensure that potential estimates of burden of disease are reported in adherence with the guidelines for accurate and transparent health estimates reporting (GATHER) (The GATHER Working Group, 2016; Stevens et al., 2016), WHO and ILO require a systematic review of studies on the prevalence of occupational exposure to dusts and/or fibres (Systematic Review 1), as well as a second systematic review and meta-analysis of studies with estimates of the relative effect of occupational exposure to dusts and/or fibres on the prevalence of, incidence of and mortality from pneumoconiosis, compared with the theoretical minimum risk exposure level (Systematic Review 2). The theoretical minimum risk exposure level is the exposure level that would result in the lowest possible population risk, even if it is not feasible to attain this exposure level in practice (Murray et al., 2004). These data and effect estimates should be tailored to serve as parameters for estimating the burden of pneumoconiosis from occupational exposure to asbestos, silica and/or coal dust in the WHO/ILO joint methodology. 
Apart from one recent systematic review assessing exposure to pure coal dust and the risk of interstitial lung diseases (Beer et al., 2017), we have not identified any previous systematic reviews on occupational exposure to dusts and/or fibres and on the effect of dusts and/or fibres on pneumoconiosis. We are aware that asbestos, silica and coal dust can lead to diseases other than pneumoconiosis (e.g. different types of cancer and chronic obstructive pulmonary disease), but these will not be investigated in our systematic reviews.

Work in the informal economy may lead to different exposures and exposure effects than does work in the formal economy. The informal economy is defined as: "all economic activities by workers and economic units that are - in law or in practice - not covered or insufficiently covered by formal arrangements", but excluding "illicit activities, in particular the provision of services or the production, sale, possession or use of goods forbidden by law, including the illicit production and trafficking of drugs, the illicit manufacturing of and trafficking in firearms, trafficking in persons, and money laundering, as defined in the relevant international treaties" (104th International Labour Conference, 2015). Therefore, we consider the formality of the economy reported in studies included in both systematic reviews.

\subsection{Description of risk factors}

We will review occupational exposure to three different types of dusts and/or fibres as separate risk factors for pneumoconiosis: (i) asbestos; (ii) silica; and (iii) coal dust. We define coal dust as either dust from coal mining or dust from pure coal. Coal dust from coal mining may contain either or a combination of different types of coal, various silicates and asbestos fibres, depending on the specific mineral composition of the mined substance. There are workers with exposure to coal dust only, such as those working in (bulk) transportation (e.g. bulk ports) and who use coal at work (e.g. coke ovens, electricity power plants and other industries using coal as ground material or power source). However, the most numerous occupational groups with exposure to coal dust include workers involved in excavating mineral dust (e.g. excavators, haulage, maintenance and surface workers) and those working at the seam of coal mines. The definition of the risk factors, the risk factor levels and their theoretical minimum risk exposure level are presented in Table 1 . We define the risk factor as any occupational exposure by inhalation to dusts and/or fibres in the air. We assume a theoretical minimum risk exposure level of no occupational exposure. Since the theoretical minimum risk exposure level is usually set empirically based on the causal epidemiological evidence, we will change the assumed level as evidence suggests. If several studies report exposure levels differing from the two standard levels we define here, then, if possible, we will convert the reported levels to the standard levels and, if not possible, we will report analyses on these alternate exposure levels as supplementary information in the systematic review.

\subsection{Description of outcomes}

In Systematic Review 2, we will review four different outcomes: (i) asbestosis (as the outcome of occupational exposure to asbestos); (ii) silicosis (from occupational exposure to silica); (iii) coal workers' pneumoconiosis (from occupational exposure to coal dust); and (iv) unspecified pneumoconiosis (from asbestos, silica and/or coal dust).
Table 2

Pairs of occupational risk factors and pneumoconiosis included in this review.

\begin{tabular}{lll}
\hline Pair & Risk factor & Disease or health problem \\
\hline 1 & Occupational exposure to asbestos & Asbestosis \\
2 & Occupational exposure to silica & Silicosis \\
3 & Occupational exposure to coal dust & Coal worker pneumoconiosis \\
4 & Occupational exposure to asbestos, silica & Unspecified pneumoconiosis \\
& and/or coal dust & \\
\hline
\end{tabular}

We will consider prevalence of, incidence of and mortality from the conditions. The WHO Global Health Estimates groups outcomes into standard burden of disease categories (World Health Organization, 2017), based on standard codes from the International Statistical Classification of Diseases and Related Health Problems 10th Revision (ICD-10) (World Health Organization, 2015). The relevant WHO Global Health Estimates category for Systematic Review 2 is "II.I.3 Other respiratory diseases" (World Health Organization, 2017). In line with the WHO Global Health Estimates, we define the four types of pneumoconiosis covered in Systematic Review 2 as asbestosis (ICD-10 code: J61), silicosis (J62), coal worker pneumoconiosis (J60), and unspecified pneumoconiosis (J64). Outcomes classified using categories and codes from previous ICD versions will be mapped to ICD-10, and eligible outcomes will be included. Table 2 presents the included pairs of occupational risk factors and pneumoconiosis types. Table 3 presents each disease or health problem included in the WHO Global Health Estimates category and whether it is included in this review. This review covers only part of the entire burden of disease envelope of the relevant WHO Global Health Estimates category.

\subsection{How the risk factor may impact the outcome}

Official health estimates of the burden of disease attributable to an occupational risk factor require a sufficient level of scientific consensus that the risk factor causes the disease (Stevens et al., 2016). Mechanistic and experimental evidence suggests that exposure to asbestos, silica and/or coal dust causes pneumoconiosis through fibrotic degeneration of lung tissue through different mechanisms, including inflammation, oxidative stress, iron complexation and apoptosis. Animal studies also support a causal effect of asbestos, silica and/or coal dust on pneumoconiosis.

Fig. 1 presents the logic model for our systematic review of the causal relationship between occupational exposure to dusts and fibres and pneumoconiosis. This logic model is an a priori, process model (Rehfuess et al., 2018) that seeks to capture complexity of the risk factor-outcome causal relationship (Anderson et al., 2011). As shown in the logic model, asbestos and silica can be primary exposures, but also be co-exposure to coal dust exposure (e.g. in coal mining). Asbestos, silica and coal dust can occur in combination with other/unknown risk factors and then lead to coalworker or unspecified pneumoconiosis. Important confounders are age, gender, turberculosis and socioeconomic position. Note that in addition to a confounder, tuberculosis could also be a consequence of pneumoconiosis. Country, age, gender, socioeconomic position, industrial sector, occupational and formality of work could potentially modify the causal effect.

Table 1

Definitions of risk factors, risk factor levels and the minimum risk exposure level.

\begin{tabular}{|c|c|c|c|}
\hline & Risk factor 1 & Risk factor 2 & Risk factor 3 \\
\hline Risk factor & Occupational exposure to asbestos & Occupational exposure to silica & Occupational exposure to coal dust \\
\hline Risk factor levels & $\begin{array}{l}\text { 1. No occupational exposure to asbestos } \\
\text { 2. Any occupational exposure to asbestos }\end{array}$ & $\begin{array}{l}\text { 1. No occupational exposure to silica } \\
\text { 2. Any occupational exposure to silica }\end{array}$ & $\begin{array}{l}\text { 1. No occupational exposure to coal dust } \\
\text { 2. Any occupational exposure to coal dust }\end{array}$ \\
\hline Theoretical minimum risk exposure level & No occupational exposure to asbestos & No occupational exposure to silica & No occupational exposure to coal dust \\
\hline
\end{tabular}


Table 3

ICD-10 codes and disease and health problems covered by the WHO burden of disease category II.I.3 Other respiratory diseases and their inclusion in this review.

\begin{tabular}{|c|c|c|}
\hline ICD-10 code & Disease or health problem & Included in this review \\
\hline J30-J39 & Other diseases of upper respiratory tract & No \\
\hline $\mathrm{J} 47$ & Bronchiectasis & No \\
\hline $\mathrm{J} 60$ & Coal worker pneumoconiosis & Yes \\
\hline $\mathrm{J} 61$ & Pneumoconiosis due to asbestos and other mineral fibres & Yes \\
\hline $\mathrm{J} 62$ & Pneumoconiosis due to dust containing silica & Yes \\
\hline J63 & Pneumoconiosis due to other inorganic dusts & No \\
\hline J64 & Unspecified pneumoconiosis & Yes \\
\hline J65 & Pneumoconiosis associated with tuberculosis & No \\
\hline J66 & Airway disease due to specific organic dust & No \\
\hline J67 & Hypersensitivity pneumonitis due to organic dust & No \\
\hline $\mathrm{J} 68$ & Respiratory conditions due to inhalation of chemicals, gases, fumes and vapours & No \\
\hline J69 & Pneumonitis due to solids and liquids & No \\
\hline J70 & Respiratory conditions due to other external agents & No \\
\hline J80-J84 & Other respiratory diseases principally affecting the interstitium & No \\
\hline J85-J86 & Suppurative and necrotic conditions of lower respiratory tract & No \\
\hline J90-J94 & Other diseases of pleura & No \\
\hline J95-J99 & Other diseases of the respiratory system & No \\
\hline
\end{tabular}

\subsubsection{Evidence from mechanistic or experimental studies}

Pneumoconiosis is defined as "a diagnosable disease of the lungs produced by the inhalation of inorganic dust, the term 'dust' being understood [to be] particulate matter in the solid phase but excluding living organisms" (Freundlich and Bragg, 1997) In particular, silica, asbestos and coal dust are known risk factors for pneumoconiosis. Their role in the etiology of pneumoconiosis is supported by the relationship of typical radiological and clinical findings with exposure for at least several years (as evaluated by a detailed occupational history). Performing lung biopsies solely for diagnoses of pneumoconiosis are not appropriate. However, if lung specimens are available from other investigations, then detection of silica, coal dust and/or asbestos fibres in lung tissue, combined with the typical pathological lesions, prove pneumoconiosis (Chong et al., 2006; Abraham, 2006). Although the mechanism of the diseases is still not completely elucidated, causality for silica, asbestos and coal dust is demonstrated by post mortem pathohistological evaluation of lung tissue for silicosis, asbestosis and coalworker pneumoconiosis (Ndlovu et al., 2017; Ndlovu et al., 2016; Nelson et al., 2010; Naidoo et al., 2005; Corbett et al., 1999; Hnizdo et al., 1993; Nelson et al., 2011; Murray et al., 1996). The presence of slate-grey to dense black silica dust-laden macrophages (sometimes visible silicate crystals), asbestos fibres and asbestos bodies and pigment-laden macrophages, and anthracitic pigmentation (coal dust) are evidence for exposure, but they are not sufficient for the diagnosis of pneumoconiosis (Landrigan, 2016). On the other hand, the absence of these lesions does not exclude a diagnosis of pneumoconiosis (Landrigan, 2016). Studies in rodent disease models confirm a role of crystalline silica and asbestos fibres in the etiology of silicosis and asbestosis, respectively. Dose-related effects were observed in disease models of acute and chronic silicosis in rats (Aalto et al., 1989; Reiser et al., 1983; Reiser et al., 1982; Le Maho et al., 1984). In the fibrotic degeneration of lung tissue, different mechanisms have been suggested, including inflammation, oxidative stress, iron complexation and apoptosis (Langley et al., 2011; Shimizu et al., 2015). Mitogen-activated protein kinase (MAPK) pathways are activated by asbestos fibres and promote epithelial apoptosis. In this pathway, extracellular signalregulated kinases (ERK1/2) affect downstream tumour necrosis factor alpha (TNFa) (Mossman et al., 2007). When exposing knockout mice lacking a TNF $\alpha$ response to chrysotile asbestos, resistance to asbestosis was observed (Sullivan et al., 2005). For silicosis, coal miner pneumoconiosis and unspecific pneumoconiosis, the role of oxidative stress, inflammation and apoptosis was well synthesized in a 2006 review of evidence (Gulumian et al., 2006). A review of the role of genotype and phenotype susceptibility studied in bronchoalveolar lavage obtained

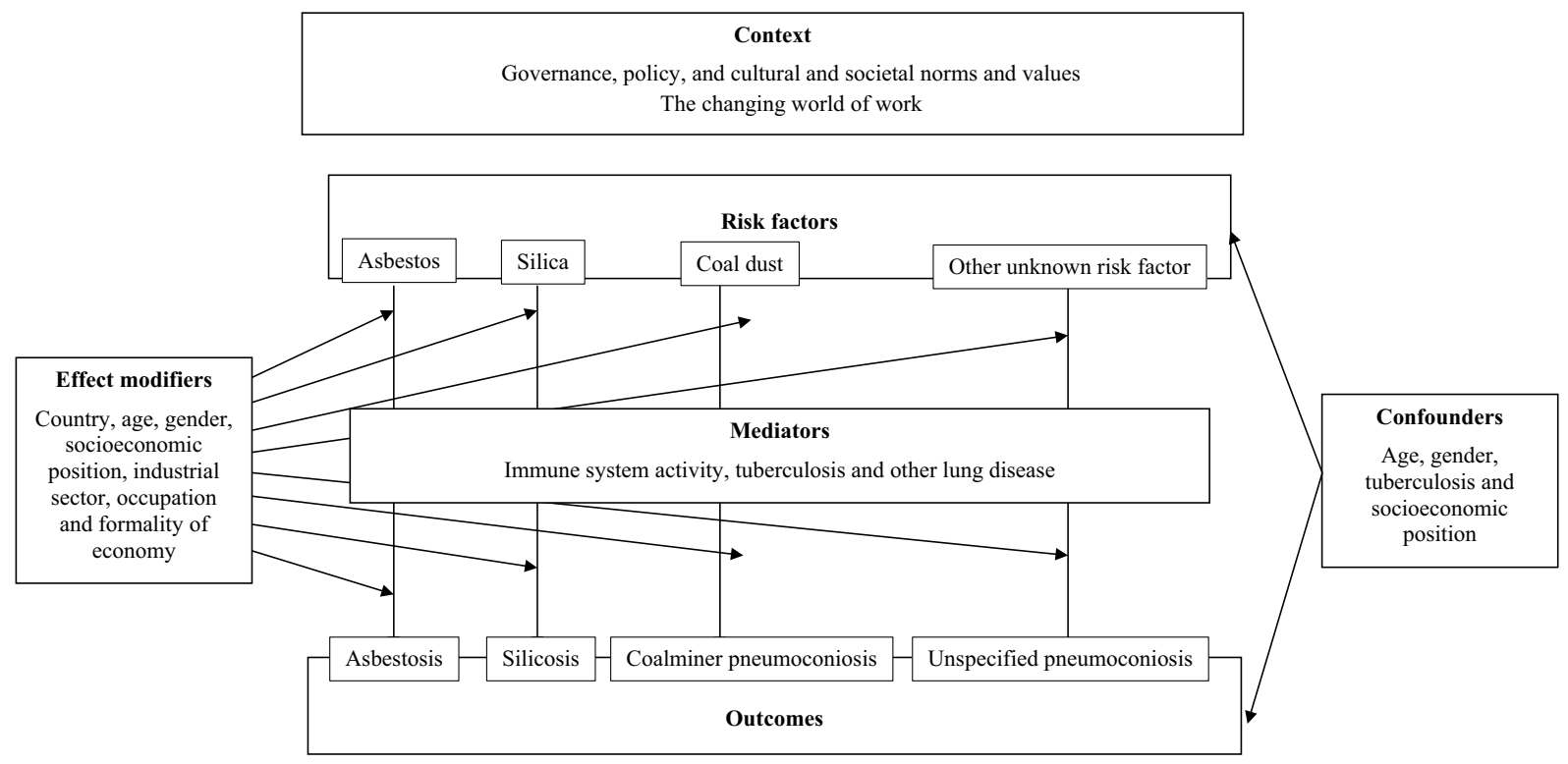

Fig. 1. Logic model of the potential causal relationship between occupational exposure to dusts and/or fibres and pneumoconiosis. 
from patients diagnosed with coal miner pneumoconiosis is also available elsewhere (Borm and Schins, 2001).

\section{Objectives}

1. Systematic Review 1: To systematically review quantitative studies of any design on the prevalence of occupational exposure to dusts and/or fibres of: (i) asbestos; (ii) silica; and (iii) coal during the years 1960 to 2018 among the working-age population, disaggregated by country, sex, age and industrial sector occupation.

2. Systematic Review 2: To systematically review and meta-analyse randomized controlled trials, cohort studies, case-control studies and other non-randomized intervention studies including estimates of the relative effect of any occupational exposure to dusts and/or fibres (i.e. asbestos, silica and coal dust) on pneumoconiosis (i.e. asbestosis, silicosis, coal worker pneumoconiosis and unspecified pneumoconiosis) in any year among the working-age population, compared with the minimum risk exposure level of no exposure.

\section{Methods}

We will apply the Navigation Guide (Woodruff and Sutton, 2014) systematic review methodology for systematic reviews in occupational and environmental health as our guiding methodological framework, wherever feasible. The guide applies established systematic review methods from clinical medicine, including standard Cochrane Collaboration methods for systematic reviews of interventions, to the field of occupational and environmental health to ensure systematic and rigorous evidence synthesis on occupational and environmental risk factors that reduces bias and maximizes transparency (Woodruff and Sutton, 2014). The need for further methodological development and refinement of the relatively novel Navigation Guide has been acknowledged (Woodruff and Sutton, 2014).

Systematic Review 1 may not map well to the Navigation Guide framework (Fig. 1 on page 1009 in (Lam et al., 2016a)), which is tailored to hazard identification and risk assessment. Nevertheless, steps 1-6 for the stream on human data can be applied to systematically review exposure to risk factors. Systematic Review 2 maps more closely to the Navigation Guide framework, and we will conduct steps 1-6 for the stream on human data, but not conduct any steps for the stream on non-human data, although we will briefly summarize narratively the evidence from non-human data that we are aware of.

We have registered the protocol in PROSPERO under CRD42018084131. This protocol adheres with the preferred reporting items for systematic review and meta-analysis protocols statement (PRISMA-P) (Moher et al., 2015; Shamseer et al., 2015), with the abstract adhering with the reporting items for systematic reviews in journal and conference abstracts (PRISMA-A) (Beller et al., 2013). Any modification of the methods stated in the present protocol will be registered in PROSPERO and reported in the systematic review itself. Systematic Review 1 will be reported according to the GATHER guidelines (Stevens et al., 2016), and Systematic Review 2 will be reported according to the preferred reporting items for systematic review and meta-analysis statement (PRISMA) (Liberati et al., 2009). Our reporting of all parameters for estimating the burden of pneumoconiosis from occupational exposure to dusts and/or fibres in the systematic review will adhere with the requirements of the GATHER guidelines (Stevens et al., 2016), because the WHO/ILO burden of disease estimates that may be produced consecutive to the systematic review must also adhere to these reporting guidelines.

\subsection{Systematic review 1}

\subsubsection{Eligibility criteria}

The population, exposure, comparator and outcome (PECO) (Woodruff and Sutton, 2014) criteria are described below.
3.1.1.1. Types of populations. We will include studies of workers working-age ( $\geq 15$ years) in the formal and informal economy. Studies of children (aged $<15$ years) and unpaid domestic workers will be excluded. Participants residing in any WHO and/or ILO Member State and workers in any industrial setting and occupation will be included. We note that occupational exposure to dusts and/or fibres may potentially have further population reach (e.g. across generations for workers of reproductive age, through take-home exposures to family members, and through the release of dusts and/ or fibres from the workplace into the community) and acknowledge that the scope of our systematic reviews will not be able capture these populations and impacts on them. Appendix A provides a complete, but briefer overview of the PECO criteria.

3.1.1.2. Types of exposures. We will include studies that define occupational exposure to dusts and/or fibres in accordance with our standard definition (Table 1). For pneumoconiosis, cumulative exposure may be the most biologically relevant exposure metric, but we will also prioritize a non-cumulative exposure metric, because we believe that insufficient cumulative exposure data currently exist to enable burden of disease estimation. We will review evidence separately for dusts and/or fibres from (i) asbestos, (ii) silica and (iii) coal dust.

We will include studies on the prevalence of occupational exposure to the respective risk factor, if it is disaggregated by country, sex (two categories: female, male), age group (ideally in 5-year age bands, such as 20-24 years) and industrial sector (e.g. International Standard Industrial Classification of All Economic Activities, Revision 4 [ISIC Rev. 4]) (United Nations, 2008) or occupation (as defined, for example, by the International Standard Classification of Occupations 1988 [ISCO-88] (International Labour Organization, 1987) or 2008 [ISCO-08] (International Labour Organization, 2012)). Criteria may be revised in order to identify optimal data disaggregation to enable subsequent estimation of the burden of disease.

We shall include studies with exposure data for the years 1960 to April 30th 2018, as WHO and ILO aim to estimate burden of disease as of 2015. We will include data beyond 2015, because these can be helpful for modelling exposures in the year 2015. We will summarize the body of evidence on the lag time between occupational exposure to dusts and/or fibres and pneumoconiosis, but assume new onset pneumoconiosis to occur up to 10 years after exposure to dusts and/or fibres (Haibing et al., 2006).

We will include only objective measurements of occupational exposure to eligible dusts and/or fibres, such as quantitative samples of dust and/or fibres collected by an expert using appropriate technologies. Subjective measures will be excluded, such as self-reports from workers, workplace administrators or managers. We will include studies with measures from any data source, including registry data.

The exposure parameter should match the one used in Systematic Review 2 or can be converted to match it.

3.1.1.3. Types of comparators. There will be no comparator, because we will review risk factor prevalence only.

3.1.1.4. Types of outcomes. Exposure to the occupational risk factor (i.e. dusts and/or fibres).

3.1.1.5. Types of studies. We will include quantitative studies of any design, including cross-sectional studies. These studies must be representative of the relevant industrial sector, occupational group or national population. We will exclude qualitative, modelling and case studies, as well as non-original studies without quantitative data (e.g. letters, commentaries and perspectives).

Records written in any language will be included. If a record is written in a language other than those spoken by the authors of this review or those of other reviews in the series (Hulshof et al., 2018; 
Tenkate et al., 2018; John et al., 2018; Teixeira et al., 2018; Li et al., 2018; Descatha et al., 2018; Rugulies et al., 2018; Pachito et al., 2018) (i.e. Arabic, Bulgarian, Chinese, Danish, Dutch, English, French, Finnish, German, Hungarian, Italian, Japanese, Norwegian, Portuguese, Russian, Spanish, and Swedish), then the record will be translated into English. Published and unpublished studies will be included.

Studies conducted using unethical practices will be excluded from the review (e.g., studies that deliberately exposed humans to a known risk factor to human health).

3.1.1.6. Types of effect measures. We will include studies with a measure of the prevalence of occupational exposure to dusts and/or fibres.

\subsubsection{Information sources and search}

3.1.2.1. Electronic academic databases. We (DM, VS) will at a minimum search the five following electronic academic databases:

1. Ovid Medline with Daily Update (1946 to 30 April 2018).

2. PubMed (1946 to 30 April 2018).

3. EMBASE (1974 to 30 April 2018).

4. Web of Science with inclusion of three databases: Science Citation Index Expanded (1900 to 30 April 2018); Social Sciences Citation Index (1956 to 30 April 2018); and Arts and Humanities Citation Index (1975 to 30 April 2018).

5. OSH UPDATE with inclusion of three databases: CISDOC (1974 to 30 April 2018); HSELINE (1977 to 30 April 2018); and NIOSHTIC-2 (1977 to 30 April 2018).

The Ovid Medline search strategy for Systematic Review 1 is presented in Appendix B. We will perform searches in electronic databases operated in the English language using a search strategy in the English language. We will adapt the search syntax to suit the other electronic academic and grey literature databases. When we are nearing completion of the review, we will search the PubMed database for the most recent publications (e.g., e-publications ahead of print) over the last six months. Any deviation from the planned search strategy in the actual search strategy will be documented.

3.1.2.2. Electronic grey literature databases. DM and VS will at a minimum search the two following electronic academic databases:

1. OpenGrey (http://www.opengrey.eu/).

2. Grey Literature Report (http://greylit.org/).

3.1.2.3. Internet search engines. We (DM, VS) will also search the Google (www.google.com/) and Google Scholar (www.google.com/ scholar/) Internet search engines and screen the first 100 hits for potentially relevant records.

3.1.2.4. Organizational websites. The websites of the following seven international organizations and national government departments will be searched by DM and VS:

1. International Labour Organization (www.ilo.org/).

2. World Health Organization (www.who.int).

3. European Agency for Safety and Health at Work (https://osha. europa.eu/en).

4. Eurostat (www.ec.europa.eu/eurostat/web/main/home).

5. China National Knowledge Infrastructure (http://www.cnki.net/).

6. Finnish Institute of Occupational Health (https://www.ttl.fi/en/).

7. United States National Institute of Occupational Safety and Health (NIOSH), using the NIOSH data and statistics gateway (https:// www.cdc.gov/niosh/data/).

3.1.2.5. Hand-searching and expert consultation. We (DM, VS) will hand- search for potentially eligible studies in:

- Reference lists of previous systematic reviews.

- Reference lists of all included study records.

- Study records published over the past 24 months in the three peerreviewed academic journals from which we obtain the largest number of included studies.

- Study records that have cited an included study record (identified in the Web of Science citation database).

- Collections of the review authors.

Additional experts will be contacted with a list of included studies, with the request to identify potentially eligible additional studies.

\subsubsection{Study selection}

Study selection will be carried out with the Rayyan Systematic Reviews Web App (Ouzzani et al., 2016) or DistillerSR (EvidencePartner, 2017). All study records identified in the search will be downloaded and duplicates will be identified and deleted. Afterwards, at least two review authors (DM, VS) will independently screen titles and abstracts (step 1) and then full texts (step 2) of potentially relevant records. A third review author (PS) will resolve any disagreements between the two review authors. If a study record identified in the literature search was authored by a review author assigned to study selection or if an assigned review author was involved in the study, then the record will be re-assigned to another review author for study selection. In the systematic review, we will document the study selection in a flow chart, as per GATHER guidelines (Stevens et al., 2016).

\subsubsection{Data extraction and data items}

A data extraction form will be developed and piloted until there is convergence and agreement among data extractors. At a minimum, two review authors (DM, VS) will independently extract the data on exposure to dusts and/or fibres, disaggregated by country, sex, age and industrial sector or occupation. A third review author (PS) will resolve conflicting extractions. At a minimum, we will extract data on study characteristics (including study authors, study year, study country, participants, exposure and outcome), study design (including study type and measurement of the risk factor), risk of bias (including missing data, as indicated by response rate and other measures) and study context. The estimates of the proportion of the population exposed to the occupational risk factor from included studies will be entered into and managed with the Review Manager, Version 5.3 (RevMan 5.3) (Review Manager (RevMan), 2014) or DistillerSR (EvidencePartner, 2017) softwares.

We will also extract data on potential conflict of interest in included studies, including the financial disclosures and funding sources of each author and their affiliated organization. We will use a modification of a previous method to identify and assess undisclosed financial interests (Forsyth et al., 2014). Where no financial disclosure/conflict of interest is provided, we will search declarations of interest both in other records from this study published in the 36 months prior to the included study record and in other publicly available repositories (Mandrioli et al., 2016; International Committee of Medical Journal Editors, 2010).

We will request missing data from the principal study author by email or phone, using the contact details provided in the principal study record. If no response is received, we will follow up twice via email, at two and four weeks.

\subsubsection{Risk of bias assessment}

Generally agreed methods (i.e. framework plus tool) for assessing risk of bias do not exist for systematic reviews for burden of disease studies and those of input data for health estimates (The GATHER Working Group, 2016), prevalence studies in general (Munn et al., 2014), and prevalence studies of occupational and/or environmental risk factors specifically (Krauth et al., 2013; Mandrioli and Silbergeld, 
2016; Vandenberg et al., 2016). None of the five standard risk of bias assessment methods for systematic reviews in occupational and occupational and environmental health (Rooney et al., 2016) are applicable to assessing prevalence studies. The Navigation Guide does not support checklist approaches (Munn et al., 2014; Hoy et al., 2012) for assessing risk of bias in prevalence studies.

We will use a modified version of the Navigation Guide risk of bias tool that we developed specifically for Systematic Review 1 (Appendix C). We will assess risk of bias on the levels of the individual study and the entire body of evidence. As per our preliminary tool, we will assess risk of bias along five domains: (i) selection bias; (ii) performance bias; (iii) misclassification bias; (iv) conflict of interest; and (v) other biases. Risk of bias will be: "low"; "probably low"; "probably high"; "high" or "not applicable" (Lam et al., 2016a). To judge the risk of bias in each domain, we will apply our a priori instructions (Appendix C).

All risk of bias assessors (DM, VS, PS) will trial the tool until they synchronize their understanding and application of each risk of bias domain, considerations and criteria for ratings. At least two study authors (DM, VS) will then independently judge the risk of bias for each study by outcome, and a third author (PS) will resolve any conflicting judgments. We will present the findings of our risk of bias assessment for each eligible study in a standard 'Risk of bias' table (Higgins et al., 2011). Our risk of bias assessment for the entire body of evidence will be presented in a standard 'Risk of bias summary' figure (Higgins et al., 2011).

\subsubsection{Summary measures and synthesis of results}

We will neither produce any summary measures, nor synthesise the evidence quantitatively. The included evidence will be presented in what could be described as an 'evidence map'. All included data points from included studies will be presented, together with meta-data on the study design, number of participants, characteristics of population, setting and exposure measurement of the data point.

\subsubsection{Quality of evidence assessment}

There is no agreed method for assessing quality of evidence in systematic reviews of the prevalence of occupational and/or environmental risk factors. We will adopt/adapt from Lam et al. (Lam et al., 2016a) the latest Navigation Guide instructions for grading, including criteria (Appendix D). We will downgrade for the following five reasons from the Grading of Recommendations Assessment, Development and Evaluation (GRADE) approach: (i) risk of bias; (ii) inconsistency; (iii) indirectness; (iv) imprecision; and (v) publication bias (Schünemann et al., 2011). We will grade the evidence, using the three Navigation Guide quality of evidence ratings: "high", "moderate" and "low" (Lam et al., 2016a). Within each of the relevant reasons for downgrading, we will rate any concern per reason as "none", "serious" or "very serious". We will start at "high" for non-randomized studies and will downgrade for no concern by nil (104th International Labour Conference, 2015), for a serious concern by one grade $(-1)$, and for a very serious concern by two grades $(-2)$. We will not up-grade or down-grade the quality of evidence for the three other reasons normally considered in GRADE assessments (i.e. large effect, dose-response and plausible residual confounding and bias), because we consider them irrelevant for prevalence estimates.

All quality of evidence assessors (DM, PS, VS) will trial the application of our instructions and criteria for quality of evidence assessment until their understanding and application is synchronized. At least two study authors (DM, VS) will independently judge the quality of evidence for the entire body of evidence by outcome. A third study author (PS) will resolve conflicting judgments. In the systematic review, for each outcome, we will present our assessments of the risk for each GRADE domain, as well as an overall GRADE rating.

\subsubsection{Strength of evidence assessment}

To our knowledge, no agreed method exists for rating strength of evidence in systematic reviews of prevalence studies. We (DM, PS, VS) will rate the strength of the evidence for use as input data for estimating national-level exposure to the risk factor. Our rating will be based on a combination of the following four criteria: (i) quality of the entire body of evidence; (ii) population coverage of evidence (WHO regions and countries); (iii) confidence in the entire body of evidence; and (iv) other compelling attributes of the evidence that may influence certainty. We will rate the strength of the evidence as either "potentially sufficient" or "potentially inadequate" for use as input data (Appendix E).

\subsection{Systematic review 2}

\subsubsection{Eligibility criteria}

The PECO (Woodruff and Sutton, 2014) criteria are described below.

3.2.1.1. Types of populations. We will include studies of working-age workers ( $\geq 15$ years) in the formal and informal economy. Studies of children ( $<15$ years) and unpaid domestic workers will be excluded. Participants residing in any WHO and/or ILO Member State and workers in any industrial setting and occupation will be included. We note that occupational exposure to dusts and/or fibres may potentially have further population reach (e.g. across generations for workers of reproductive age, through take-home exposures to family members, and through the release of dusts and/or fibres from the workplace into the community) and acknowledge that the scope of our systematic reviews will not be able capture these populations and impacts on them. Appendix F provides an overview of the PECO criteria.

3.2.1.2. Types of exposures. We will include studies that define occupational exposure to dusts and/or fibres, in accordance with our standard definition (Table 1). We will review evidence separately for dusts and/or fibres from (i) asbestos, (ii) silica and (iii) coal dust. Studies from any year will be included.

We will include only objective measurements of occupational exposure to eligible dusts and/or fibres, such as quantitative samples of dust and/or fibres collected by an expert using appropriate technologies. Subjective measures will be excluded. We will include studies with measures from any data source.

3.2.1.3. Type of comparators. The included comparator will be participants exposed to the theoretical minimum risk exposure level (Table 1). The WHO (Murray et al., 2004) and Institute for Health Metrics and Evaluation (G. B. D. Risk Factors Collaborators, 2017) burden of disease studies on the risk factors of occupational exposure to asbestos and silica have used the same comparator. We will exclude all other comparators.

3.2.1.4. Types of outcomes. We will include studies that defined pneumoconiosis in accordance with our standard definition (Table 3). Diagnosis of asbestosis, silicosis and coal worker pneumoconiosis should be based on the following objective measures: histopathology (autopsy) of lung tissue; X-ray or computed tomography imaging (according to ILO classification); and/or other objective indicators of morphological changes. Medically certified cause of death is also included to capture mortality. If asbestosis, silicosis, and/or coal worker pneumoconiosis co-exist, we will use the classification of coal worker pneumoconiosis. Progressive massive fibrosis is a radiological and pathological characteristic that is included in our outcome. Subjective measures will be excluded.

The following measurements of pneumoconiosis will be regarded as eligible:

i) Diagnosis by a physician.

ii) Hospital discharge record.

iii) Other relevant administrative records (e.g. records of sickness 
absence or disability).

iv) Medically certified cause of death.

All other measure will be excluded from this systematic review.

3.2.1.5. Types of studies. We will include studies that investigate the effect of asbestos, silica and/or coal dust on asbestosis, silicosis and/or coal worker pneumoconiosis, and unspecified pneumoconiosis, respectively, for any years. Eligible study designs will be randomized controlled trials (including parallel-group, cluster, cross-over and factorial studies), cohort studies (both prospective and retrospective), case-control studies, and other non-randomized intervention studies (including quasi-randomized controlled trials, controlled before-after studies and interrupted time series studies). We included a broader set of observational study designs than is commonly included, because a recent augmented Cochrane Review of complex interventions identified valuable additional studies using such a broader set of study designs (Arditi et al., 2016). As we have an interest in quantifying risk (or risk assessment) and not in qualitative assessment of hazard (or hazard identification) (Barroga and Kojima, 2013), we will exclude all other study designs (e.g. uncontrolled before-and-after, cross-sectional, qualitative, modelling, case and non-original studies).

Records published in any year and any language will be included. If a record is written in a language other than those spoken by the authors of this review or those of other reviews in the series (Hulshof et al., 2018; Tenkate et al., 2018; John et al., 2018; Teixeira et al., 2018; Li et al., 2018; Descatha et al., 2018; Rugulies et al., 2018; Pachito et al., 2018), then the record will be translated into English. Published and unpublished studies will be included.

Studies conducted using unethical practices will be excluded from the review (e.g., studies that deliberately exposed humans to a known risk factor to human health).

3.2.1.6. Types of effect measures. We will include measures of the relative effect of any occupational exposure to dusts and/or fibres on the prevalence of, incidence of or mortality from pneumoconiosis, compared with the theoretical minimum risk exposure level of nonexposure. In studies with no versus any exposure, the risk estimate may be assessed based on risk estimated in low- versus high-exposed workers. We will include relative effect measures such as risk ratios and odds ratios for prevalence and mortality measures and hazard ratios for incidence and mortality measures (e.g. developed or died from pneumoconiosis). Measures of absolute effects (e.g. mean differences in risks or odds) will be converted into relative effect measures, but if conversion is impossible, they will be excluded. To ensure comparability of effect estimates and facilitate meta-analysis, if a study presents an odds ratio, then we will convert it into a risk ratio, using the guidance provided in the Cochrane Collaboration's handbook for systematic reviews of interventions (Higgins and Green, 2011).

If a study presents estimates for the effect from two or more alternative models that have been adjusted for different variables, then we will systematically prioritize the estimate from the model that we consider best adjusted, applying the lists of confounders and mediators identified in our logic model (Fig. 1). We will prioritize estimates from models adjusted for more potential confounders over those from models adjusted for fewer. For example, if a study presents estimates from a crude, unadjusted model (Model A), a model adjusted for one potential confounder (Model B) and a model adjusted for two potential confounders (Model C), then we will prioritize the estimate from Model C. We will prioritize estimates from models unadjusted for mediators over those from models that adjusted for mediators, because adjustment for mediators can introduce bias. For example, if Model A has been adjusted for two confounders, and Model B has been adjusted for the same two confounders and a potential mediator, then we will choose the estimate from Model A over that from Model B. We prioritize estimates from models that can adjust for time-varying confounders that are at the same time also mediators, such as marginal structural models (Pega et al., 2016), over estimates from models that can only adjust for timevarying confounders, such as fixed-effects models (Gunasekara et al., 2014), over estimates from models that cannot adjust for time-varying confounding. If a study presents effect estimates from two or more potentially eligible models, then we will explain specifically why we prioritized the selected model.

\subsubsection{Information sources and search}

3.2.2.1. Electronic academic databases. At a minimum, we (DM, VS) will search the six following electronic academic databases:

1. International Clinical Trials Register Platform (to 30 April 2018).

2. Ovid Medline with Daily Update (1946 to 30 April 2018).

3. PubMed (1946 to 30 April 2018).

4. EMBASE (1947 to 30 April 2018).

5. Web of Science with inclusion of three databases: Science Citation Index Expanded (1900 to 30 April 2018); Social Sciences Citation Index (1956 to 30 April 2018); Arts and Humanities Citation Index (1975 to 30 April 2018).

6. OSH UPDATE with inclusion of three databases: CISDOC (1974 to 30 April 2018); HSELINE (1977 to 30 April 2018); NIOSHTIC-2 (1977 to 30 April 2018).

The Ovid Medline search strategy for Systematic Review 2 is presented in Appendix G. We will perform searches in electronic databases operated in the English language using a search strategy in the English language. We will adapt the search syntax to suit the other electronic academic and grey literature databases. When we are nearing completion of the review, we will search the PubMed database for the most recent publications (e.g., e-publications ahead of print) over the last six months. Any deviation from the proposed search strategy in the actual search strategy will be documented.

3.2.2.2. Electronic grey literature databases. DV and VS will at a minimum search the two following electronic academic databases:

1. OpenGrey (http://www.opengrey.eu/).

2. Grey Literature Report (http://greylit.org/).

3.2.2.3. Internet search engines. We (DM, VS) will also search the Google (www.google.com/) and Google Scholar (www.google.com/ scholar/) Internet search engines and screen the first 100 hits for potentially relevant records.

3.2.2.4. Organizational websites. The websites of the following seven international organizations and national government departments will be searched by DM and VS:

1. International Labour Organization (www.ilo.org/).

2. World Health Organization (www.who.int).

3. EU-OSHA (https://osha.europa.eu/en).

4. EUROSTAT (www.ec.europa.eu/eurostat/web/main/home).

5. China National Knowledge Infrastructure (CNKI) (http://www.cnki. net/).

6. Finnish Institute of Occupational Health (https://www.ttl.fi/en/).

7. National Institute of Occupational Safety and Health (NIOSH) of the United States of America, using the NIOSH data and statistics gateway (https://www.cdc.gov/niosh/data/).

3.2.2.5. Hand-searching and expert consultation. We will hand-search for potentially eligible studies in:

- Reference lists of previous systematic reviews.

- Reference lists of all included study records.

- Study records published over the last 24 months in the three peer- 
reviewed academic journals from which we obtained the largest number of included studies.

- Study records that have cited the included studies (identified in the Web of Science citation database).

- Collections of the review authors.

Additional experts will be contacted with a list of included studies, with the request to identify potentially eligible additional studies.

\subsubsection{Study selection}

Study selection will be carried out with the Rayyan Systematic Reviews Web App (Ouzzani et al., 2016) or DistillerSR (EvidencePartner, 2017). All study records identified in the search will be downloaded and duplicates will be identified and deleted. Afterwards, at least two review authors (DM, VS) will independently screen titles and abstracts (step 1) and then full texts (step 2) of potentially relevant records. A third review author (PS) will resolve any disagreements between the two review authors. If a study record identified in the literature search was authored by a review author assigned to study selection or if an assigned review author was involved in the study, then the record will be re-assigned to another review author for study selection. The study selection will be documented in a flow chart in the systematic review, as per PRISMA guidelines (Liberati et al., 2009).

\subsubsection{Data extraction and data items}

A data extraction form will be developed and trialled until data extractors reach convergence and agreement. At a minimum, two review authors (DM, VS) will extract data on study characteristics (including study authors, study year, study country, participants, exposure and outcome), study design (including summary of study design, comparator, epidemiological models used and effect estimate measure), risk of bias (including selection bias, reporting bias, confounding, and reverse causation) and study context (e.g. data on contemporaneous exposure to other occupational risk factors potentially relevant for deaths or other health loss from pneumoconiosis). A third review author (PS) will resolve conflicts in data extraction. Data will be entered into and managed with the RevMan 5.3 (Review Manager (RevMan), 2014) or DistillerSR (EvidencePartner, 2017) softwares.

We will also extract data on potential conflict of interest in included studies. For each author and affiliated organization of each included study record, we will extract their financial disclosures and funding sources. We will use a modification of a previous method to identify and assess undisclosed financial interest of authors (Forsyth et al., 2014). Where no financial disclosure or conflict of interest statements are available, we will search the name of all authors in other study records gathered for this study and published in the prior 36 months and in other publicly available declarations of interests (Mandrioli et al., 2016; International Committee of Medical Journal Editors, 2010).

We will request missing data from the principal study author by email or phone, using the contact details provided in the principal study record. If we do not receive a positive response from the study author, we will send follow-up emails twice, at two and four weeks.

\subsubsection{Risk of bias assessment}

Standard risk of bias tools does not exist for systematic reviews for hazard identification in occupational and environmental health, nor for risk assessment. The five methods specifically developed for occupational and environmental health are for either or both hazard identification and risk assessment, and they differ substantially in the types of studies (randomized, observational and/or simulation studies) and data (e.g. human, animal and/or in vitro) they seek to assess (Rooney et al., 2016). However, all five methods, including the Navigation Guide (Lam et al., 2016a), assess risk of bias in human studies similarly (Rooney et al., 2016).

The Navigation Guide was specifically developed to translate the rigour and transparency of systematic review methods applied in the clinical sciences to the evidence stream and decision context of occupational and environmental health (Woodruff and Sutton, 2014), which includes workplace environment exposures and associated health outcomes. The guide is our overall organizing framework, and we will also apply its risk of bias assessment method in Systematic Review 2. The Navigation Guide risk of bias assessment method builds on the standard risk of bias assessment methods of the Cochrane Collaboration (Higgins et al., 2011) and the US Agency for Healthcare Research and Quality (Viswanathan et al., 2008). Some further refinements of the Navigation Guide method may be warranted (Goodman et al., 2017), but it has been successfully applied in several completed and ongoing systematic reviews (Johnson et al., 2014; Koustas et al., 2014; Lam et al., 2014; Vesterinen et al., 2014; Johnson et al., 2016; Lam et al., 2016b; Lam et al., 2017; Lam et al., 2016c). In our application of the Navigation Guide method, we will draw heavily on one of its latest versions, as presented in the protocol for an ongoing systematic review (Lam et al., 2016a). Should a more suitable method become available, however, we may switch to it.

We will assess risk of bias on the levels of the individual study and the entire body of evidence. The nine risk of bias domains included in the Navigation Guide risk of bias assessment tool for human studies are: (i) source population representation; (ii) blinding; (iii) exposure assessment; (iv) outcome assessment; (v) confounding; (vi) incomplete outcome data; (vii) selective outcome reporting; (viii) conflict of interest; and (ix) other sources of bias. While two of the earlier case studies of the Navigation Guide did not utilize "outcome assessment" as a risk of bias domain for studies of human data (Johnson et al., 2014; Koustas et al., 2014; Lam et al., 2014; Vesterinen et al., 2014), all of the subsequent reviews have included this domain (Johnson et al., 2016; Lam et al., 2016a; Lam et al., 2016b; Lam et al., 2017; Lam et al., 2016c). Risk of bias or confounding ratings will be: "low"; "probably low"; "probably high"; "high" or "not applicable" (Lam et al., 2016a). To judge the risk of bias in each domain, we will apply a priori instructions (Appendix H), which we have adopted or adapted from an ongoing Navigation Guide systematic review (Lam et al., 2016a). For example, a study will be assessed as carrying "low" risk of bias from source population representation, if we judge the source population to be described in sufficient detail (including eligibility criteria, recruitment, enrolment, participation and loss to follow-up) and the distribution and characteristics of the study sample to indicate minimal or no risk of selection effects. The risk of bias at the study level will be determined by the worst rating in any bias domain for any outcome. For example, if a study is rated as "probably high" risk of bias in one domain for one outcome and "low" risk of bias in all other domains for the same outcome and in all domains for all other outcomes, the study will be rated as having a "probably high" risk of bias overall.

All risk of bias assessors will jointly trial the application of the risk of bias criteria until they have synchronized their understanding and application of the criteria. At least two study authors (DM, VS) will independently judge the risk of bias for each study by outcome. Where individual assessments differ, a third author (PS) will resolve the conflict. In the systematic review, for each included study, we will report our study-level risk of bias assessment by domain in a standard 'Risk of bias' table (Higgins et al., 2011). For the entire body of evidence, we will present the study-level risk of bias assessments in a 'Risk of bias summary' figure (Higgins et al., 2011).

\subsubsection{Summary measures and synthesis of results}

We will conduct meta-analyses separately for estimates of the effect on prevalence, incidence and mortality. If we find two or more studies with an eligible effect estimate for one of the three risk factor-outcome pairs (see Table 2), two review authors will independently investigate the clinical heterogeneity of the studies in terms of participants (including country, sex, age and industrial sector or occupation), level of risk factor exposure, comparator and outcomes. If we find that effect estimates differ considerably by country, sex and/or age, or a 
combination of these, then we will synthesise evidence for the relevant populations defined by country, sex and/or age, or combination thereof. Differences by country could include or be expanded to include differences by country group (e.g. WHO region or World Bank income group). If we find that effect estimates are clinically homogenous across countries, genders and age groups, then we will combine studies from all of these populations into one pooled effect estimate that could be applied across all individual or combinations of countries, sexes and age groups in the WHO/ILO joint methodology.

If we judge two or more studies for the relevant country, gender and age, or combination thereof, to be sufficiently clinically homogenous to potentially be combined quantitatively using quantitative meta-analysis, then we will test the statistical heterogeneity of the studies using the $\mathrm{I}^{2}$ statistic (Higgins et al., 2003). If two or more clinically homogenous studies are found to be sufficiently statistically homogenous to be combined in a meta-analysis, we will pool the risk ratios of these studies in a quantitative meta-analysis, using the inverse variance method with a random effects model to account for cross-study heterogeneity (Higgins and Green, 2011a). The meta-analysis will be conducted in RevMan 5.3 (Review Manager (RevMan), 2014), but the data for entry into these programmes may be prepared using another recognized statistical analysis programme, e.g. Stata (Stata Cooperation, 2017). We will neither quantitatively combine data from studies with different designs (e.g. combining cohort studies with casecontrols studies), nor unadjusted and adjusted models. We will only combine studies that we judge to have a minimum acceptable level of adjustment for confounders. If quantitative synthesis is not feasible, then we will synthesise the study findings narratively and identify the estimates that we judged to be the highest quality evidence available.

\subsubsection{Additional analyses}

If we source micro-data on exposure, outcome and potential confounding variables, we may conduct meta-regressions to adjust optimally for potential confounders.

If there is evidence for differences in effect estimates by country, sex, age, industrial sector and/or occupation, or by a combination of these variables, then we will conduct sub-group analyses by the relevant variable or combination of variables, as feasible. Where both studies on workers in the informal economy and studies on workers in the formal economy are included, then we will conduct sub-group analyses by formality of economy. Findings of these sub-group analyses, if any, will be used as parameters for estimating burden of disease specifically for relevant populations defined by these variables.

We will perform a sensitivity analyses that will include only studies judged to be of "low" or "probably low" risk of bias from conflict of interest; judged to be of "low" or "probably low" risk of bias; and with documented or approximated ICD-10 diagnostic codes. We may also conduct a sensitivity analysis using an alternative meta-analytic model, namely the inverse variance heterogeneity (IVhet) model (Doi et al., 2017).

\subsubsection{Quality of evidence assessment}

We will assess quality of evidence using a modified version of the Navigation Guide quality of evidence assessment tool (Lam et al., 2016a). The tool is based on the GRADE approach (Schünemann et al., 2011) adapted specifically to systematic reviews in occupational and environmental health (Morgan et al., 2016). Should a more suitable method become available, we may switch to it.

We will assess quality of evidence for the entire body of evidence by outcome. We will adopt or adapt the latest Navigation Guide instructions for grading the quality of evidence (Lam et al., 2016a). (Appendix D). We will downgrade the quality of evidence for the following five GRADE reasons: (i) risk of bias; (ii) inconsistency; (iii) indirectness; (iv) imprecision; and (v) publication bias. If our systematic review includes ten or more studies, we will generate a funnel plot to judge concerns on publication bias. If it includes nine or fewer studies, we will judge the risk of publication bias qualitatively. To assess risk of bias from selective reporting, protocols of included studies, if any, will be screened to identify instances of selective reporting.

We will grade the evidence, using the three Navigation Guide standard quality of evidence ratings: "high", "moderate" and "low" (Lam et al., 2016a). Within each of the relevant domains, we will rate the concern for the quality of evidence, using the ratings "none", "serious" and "very serious". As per the Navigation Guide, we will start at "high" for randomized studies and "moderate" for observational studies. Quality will be downgraded for no concern by nil grades, for a serious concern by one grade $(-1)$ and for a very serious concern by two grades $(-2)$. We will up-grade the quality of evidence for the following other reasons: large effect, dose-response and plausible residual confounding and bias. For example, if we have a serious concern for risk of bias in a body of evidence consisting of observational studies $(-1)$, but no other concerns, and there are no reasons for upgrading, then we will downgrade its quality of evidence by one grade from "moderate" to "low".

\subsubsection{Strength of evidence assessment}

We will apply the standard Navigation Guide methodology (Lam et al., 2016a) to rate the strength of the evidence. The rating will be based on a combination of the following four criteria: (i) quality of the body of evidence; (ii) direction of the effect; (iii) confidence in the effect; and (iv) other compelling attributes of the data that may influence our certainty. The ratings for strength of evidence for the effect of occupational exposure to dusts and/or fibres on pneumoconiosis will be "sufficient evidence of toxicity/harmfulness", "limited of toxicity/ harmfulness", "inadequate of toxicity/harmfulness" and "evidence of lack of toxicity/harmfulness" (Appendix I).

\section{Financial support}

All authors are salaried staff members of their respective institutions. The publication was prepared with financial support from the WHO cooperative agreement with the Centres for Disease Control and Prevention National Institute for Occupational Safety and Health of the United States of America on implementing Resolution WHA 60.26 "Workers' Health: Global Plan of Action (Grant 1 E11 OH0010676-02).

\section{Sponsors}

The sponsors of these systematic reviews are the World Health Organization and the International Labour Organization.

\section{Author contributions}

II, NL, FP and APÜ had the idea for the systematic review. II, NL, FP and YU gathered the review team. FP led and all authors contributed to the development of the standard methodology for all systematic reviews in the series. FP led and all authors contributed to the development and writing of the standard template for all protocols in the series. DM, PS and VS are the lead reviewers of this systematic review. DM, PS and VS wrote the first draft of this protocol, using the protocol template prepared by FP, and BA, CC, WC, AF, LG, TG, II, SMR, DM, FM, BN, FP, APÜ, DS, VS, YU, SvdM and MZ made substantial contributions to the revisions of the manuscript. The search strategy was developed and piloted by VS in collaboration with a research librarian. VS and FP are experts in epidemiology, PS is an occupational toxicologist and occupational hygienist and DM and FP are experts in systematic review methodology. FP coordinated all inputs from WHO, ILO and external experts and ensured consistency across the systematic reviews of the series. DM, PS and VS are the guarantors of the systematic reviews. 


\section{Acknowledgments}

We thank research librarian Elizabeth Bengtsen for her assistance with the search strategies. We thank Juleen Lam and Tracey Woodruff for technical training on the Navigation Guide. We are grateful to Lisa Bero, Rebecca Morgan, Susan Norris, Holger J. Schünemann, Gretchen Stevens, Patrice Sutton and Tracey Woodruff for their feedback on the methods for this protocol. Tim France technically edited the manuscript. We thank Paul Whaley and Tim Driscoll for their editorial guidance. The authors alone are responsible for the views expressed in this article and they do not necessarily represent the views, decisions or policies of the institutions with which they are affiliated.

\section{Appendix A. Supplementary data}

Supplementary data to this article can be found online at https:// doi.org/10.1016/j.envint.2018.06.005.

\section{References}

104th International Labour Conference, 2015. Transition from the Informal to the Formal Economy (Recommendation No. 204). International Labour Organization, Geneva.

Aalto, M., Kulonen, E., Pikkarainen, J., 1989. Isolation of silica-dependent protein from rat lung with special reference to development of fibrosis. Br. J. Exp. Pathol. 70 (2), 167.

Abraham, J.L., 2006. Analysis of Fibrous and Non-fibrous Particles. Environmental and Occupational Medicine, 4th edition. Lippincott Williams and Wilkins, pp. 277-297.

Akaoka, K., McKendry, I., Saxton, J., Cottle, P.W., 2017. Impact of coal-carrying trains on particulate matter concentrations in South Delta, British Columbia, Canada. Environ. Pollut. (Barking, Essex:1987) 223, 376-383.

Anderson, L.M., Petticrew, M., Rehfuess, E., et al., 2011. Using logic models to capture complexity in systematic reviews. Res. Synth. Methods 2 (1), 33-42.

Arditi, C., Burnand, B., Peytremann-Bridevaux, I., 2016. Adding non-randomised studies to a Cochrane review brings complementary information for healthcare stakeholders: an augmented systematic review and meta-analysis. BMC Health Serv. Res. 16 (1), 598.

Barroga, E.F., Kojima, T., 2013. Research study designs: an appraisal for peer reviewers and science editors. Eur. Sci. Ed. 2013, 44-45.

Beer, C., Kolstad, H.A., Sondergaard, K., et al., 2017. A systematic review of occupational exposure to coal dust and the risk of interstitial lung diseases. Eur. Clin. Respir. J. 4 (1), 1264711.

Beller, E.M., Glasziou, P.P., Altman, D.G., et al., 2013. PRISMA for abstracts: reporting systematic reviews in journal and conference abstracts. PLoS Med. 10 (4), e1001419.

Borm, P.J., Schins, R.P., 2001. Genotype and phenotype in susceptibility to coal workers' pneumoconiosis. the use of cytokines in perspective. Eur. Resp. J. Suppl. 32 (127s-33s).

Chong, S., Lee, K.S., Chung, M.J., Han, J., Kwon, O.J., Kim, T.S., 2006. Pneumoconiosis: comparison of imaging and pathologic findings. Radiographics 26 (1), 59-77.

Corbett, E.L., Murray, J., Churchyard, G.J., et al., 1999. Use of miniradiographs to detect silicosis. Comparison of radiological with autopsy findings. Am. J. Respir. Crit. Care Med. 160 (6), 2012-2017.

De Berardis, B., Incocciati, E., Massera, S., Gargaro, G., Paoletti, L., 2007. Airborne silica levels in an urban area. Sci. Total Environ. 382 (2-3), 251-258.

Descatha, A., Sembajwe, G., Baer, M., et al., 2018. WHO/ILO work-related burden of disease and injury: Protocol for systematic reviews of exposure to long working hours and of the effect of exposure to long working hours on stroke. Environ. Int. https:// doi.org/10.1016/j.envint.2018.06.016. (In press).

Doi, S.A.R., Furuya-Kanamori, L., Thalib, L., Barendregt, J.J., 2017. Meta-analysis in evidence-based healthcare: a paradigm shift away from random effects is overdue. Int. J. Evid. Based Healthc. 15 (4), 152-160.

International Committee of Medical Journal Editors, 2010. ICMJE Form for Disclosure of Potential Conflicts of Interest.

EvidencePartner, 2017. DistillerSR. Accessed from. https://www.evidencepartners.com/ products/distillersr-systematic-review-software/:EvidencePartner.

Forsyth, S.R., Odierna, D.H., Krauth, D., Bero, L.A., 2014. Conflicts of interest and critiques of the use of systematic reviews in policymaking: an analysis of opinion articles. Syst. Rev. 3, 122.

Freundlich, I.M., Bragg, D.G., 1997. A Radiologic Approach to Diseases of the Chest. 503 Lippincott Williams \& Wilkins.

G. B. D. Risk Factors Collaborators, 2017. Global, regional, and national comparative risk assessment of 84 behavioural, environmental and occupational, and metabolic risks or clusters of risks, 1990-2016: a systematic analysis for the Global Burden of Disease Study 2016. Lancet 390 (10100), 1345-1422.

Goodman, J.E., Lynch, H.N., Beck, N.B., 2017. More clarity needed in the navigation guide systematic review framework. Environ. Int. 102, 74-75.

Gulumian, M., Borm, P.J., Vallyathan, V., et al., 2006. Mechanistically identified suitable biomarkers of exposure, effect, and susceptibility for silicosis and coal-worker's pneumoconiosis: a comprehensive review. J. Toxicol. Environ. Health B Crit. Rev. 9 (5), 357-395.

Gunasekara, F.I., Richardson, K., Carter, K., Blakely, T., 2014. Fixed effects analysis of repeated measures data. Int. J. Epidemiol. 43 (1), 264-269.

Haibing, Y., Lei, Y., Junyue, Z., Jingqiong, C., 2006. Natural course of silicosis in dustexposed workers. J. Huazhong Univ. Sci. Technol. Med. Sci. 26 (2), 257-260.

Higgins, J., Green, S. (Eds.), 2011. Cochrane Handbook for Systematic Reviews of Interventions Version 5.1.0. 2011 The Cochrane Collaboration (Available from http://handbook.cochrane.org.; [updated March 2011]).

Higgins, J.P., Thompson, S.G., Deeks, J.J., Altman, D.G., 2003. Measuring inconsistency in meta-analyses. Br. Med. J. 327 (7414), 557-560.

Higgins, J., Altman, D., Sterne, J., 2011. Chapter 8: assessing risk of bias in included studies. In: Higgins, J., Green, S. (Eds.), Cochrane Handbook for Systematic Reviews of Interventions Version 510. 2011 The Cochrane Collaboration (Available from http://handbookcochraneorg; [updated March 2011]).

Hnizdo, E., Murray, J., Sluis-Cremer, G.K., Thomas, R.G., 1993. Correlation between radiological and pathological diagnosis of silicosis: an autopsy population based study. Am. J. Ind. Med. 24 (4), 427-445.

Hoy, D., Brooks, P., Woolf, A., et al., 2012. Assessing risk of bias in prevalence studies: modification of an existing tool and evidence of interrater agreement. J. Clin. Epidemiol. 65 (9), 934-939.

Hulshof, C., Colosio, C., Ivaonv, I.D., et al., 2018. WHO/ILO work-related burden of disease and injury: Protocol for systematic reviews of occupational exposure to ergonomic risk factors and of the effect of occupational exposure to ergonomic risk factors on osteoarthritis and other musculoskeletal diseases. Environ. Int (Submitted).

International Labour Organization, 1987. ISCO-88: International Standard Classification of Occupations. International Labour Organization, Geneva.

International Labour Organization, 2012. ISCO-08: International Standard Classification of Occupations. International Labour Organization, Geneva.

International Labour Organization, 2014. Safety and Health at Work: A Vision for Sustainable Prevention: XX World Congress on Safety and Health at Work 2014 Global Forum for Prevention, 24-27 August 2014, Frankfurt, Germany. International Labour Organization, Geneva.

John, S.M., Akagwu, O.C., Akparibo, I.Y., et al., 2018. WHO/ILO work-related burden of disease and injury: Protocol for systematic reviews of occupational exposure to solar ultraviolet radiation and of the effect of occupational exposure to solar ultraviolet radiation on melanoma and non-melanoma skin cancer. Environ. Int (Submitted).

Johnson, P.I., Sutton, P., Atchley, D.S., et al., 2014. The navigation guide - evidence-based medicine meets environmental health: systematic review of human evidence for PFOA effects on fetal growth. Environ. Health Perspect. 122 (10), 1028-1039.

Johnson, P.I., Koustas, E., Vesterinen, H.M., et al., 2016. Application of the navigation guide systematic review methodology to the evidence for developmental and reproductive toxicity of triclosan. Environ. Int. 92-93, 716-728.

Koustas, E., Lam, J., Sutton, P., et al., 2014. The navigation guide - evidence-based medicine meets environmental health: systematic review of nonhuman evidence for PFOA effects on fetal growth. Environ. Health Perspect. 122 (10), 1015-1027.

Krauth, D., Woodruff, T.J., Bero, L., 2013. Instruments for assessing risk of bias and other methodological criteria of published animal studies: a systematic review. Environ. Health Perspect. 121 (9), 985-992.

Lam, J., Koustas, E., Sutton, P., et al., 2014. The navigation guide - evidence-based medicine meets environmental health: integration of animal and human evidence for PFOA effects on fetal growth. Environ. Health Perspect. 122 (10), 1040-1051.

Lam, J., Sutton, P., Padula, A.M., et al., 2016a. Applying the Navigation Guide Systematic Review Methodology Case Study \#6: Association between Formaldehyde Exposure and Asthma: A Systematic Review of the Evidence: Protocol. University of California at San Francisco, San Francisco, CA.

Lam, J., Sutton, P., Halladay, A., et al., 2016b. Applying the navigation guide systematic review methodology case study \#4: association between developmental exposures to ambient air pollution and autism. PLoS ONE 21 (11), 9.

Lam, J., Koustas, E., Sutton, P., et al., 2016c. Applying the Navigation Guide: Case Study \#6. Association Between Formaldehyde Exposures and Asthma (In preparation). .

Lam, J., Lanphear, B., Bellinger, D., et al., 2017. Developmental PBDE exposure and IQ/ ADHD in childhood: a systematic review and meta-analysis. Environ. Health Perspect. 125 (8).

Landrigan, P.J., 2016. Comments on the 2014 helsinki consensus report on asbestos. Ann. Glob. Health 82 (1), 217-220.

Langley, R.J., Mishra, N.C., Peña-Philippides, J.C., et al., 2011. Fibrogenic and redoxrelated but not proinflammatory genes are upregulated in Lewis rat model of chronic silicosis. J. Toxic. Environ. Health A 74 (19), 1261-1279.

Le Maho, S., Bignon, J., Lambre, C., Jaurand, M., Masse, R., 1984. Early cellular and biochemical alveolar responses following intra-tracheal inoculation with low dose of asbestos and quartz. Arch. Immunol. Ther. Exp. 32 (1), 85-98.

Li, J., Brisson, C., Clays, E., et al., 2018. WHO/ILO work-related burden of disease and injury: Protocol for systematic reviews of exposure to long working hours and of the effect of exposure to long working hours on ischaemic heart disease. Environ. Int (Accepted).

Liberati, A., Altman, D.G., Tetzlaff, J., et al., 2009. The PRISMA statement for reporting systematic reviews and meta-analyses of studies that evaluate health care interventions: explanation and elaboration. PLoS Med. 6 (7), e1000100.

Mandrioli, D., Silbergeld, E.K., 2016. Evidence from toxicology: the most essential science for prevention. Environ. Health Perspect. 124 (1), 6-11.

Mandrioli, D., Kearns, C.E., Bero, L.A., 2016. Relationship between research outcomes and risk of Bias, study sponsorship, and author financial conflicts of interest in reviews of the effects of artificially sweetened beverages on weight outcomes: a systematic review of reviews. PLoS ONE 11 (9), e0162198.

Moher, D., Shamseer, L., Clarke, M., et al., 2015. Preferred reporting items for systematic review and meta-analysis protocols (PRISMA-P) 2015 statement. Syst. Rev. 4, 1.

Morgan, R.L., Thayer, K.A., Bero, L., et al., 2016. GRADE: assessing the quality of evidence in environmental and occupational health. Environ. Int. 92-93, 611-616. 
Mossman, B.T., Borm, P.J., Castranova, V., Costa, D.L., Donaldson, K., Kleeberger, S.R., 2007. Mechanisms of action of inhaled fibers, particles and nanoparticles in lung and cardiovascular diseases. Part. Fibre Toxicol. 4 (1), 4.

Munn, Z., Moola, S., Riitano, D., Lisy, K., 2014. The development of a critical appraisal tool for use in systematic reviews addressing questions of prevalence. Int. J. Health Policy Manag. 3 (3), 123-128.

Murray, J., Kielkowski, D., Reid, P., 1996. Occupational disease trends in black South African gold miners. An autopsy-based study. Am. J. Respir. Crit. Care Med. 153 (2), 706-710.

Murray, C.J.L., Ezzati, M., Lopez, A.D., Rodgers, A., Vander, Hoorn S., 2004. Comparative quantification of health risks: conceptual framework and methodological issues. In: Ezzati, M., Lopez, A.D., Rodgers, A., Murray, C.J.L. (Eds.), Comparative Quantification of Health Risks: Global and Regional Burden of Disease Attributable to Selected Major Risk Factors. World Health Organization, Geneva, pp. 1-39.

Naidoo, R.N., Robins, T.G., Murray, J., 2005. Respiratory outcomes among South African coal miners at autopsy. Am. J. Ind. Med. 48 (3), 217-224.

Ndlovu, N., Nelson, G., Vorajee, N., Murray, J., 2016. 38 years of autopsy findings in South African mine workers. Am. J. Ind. Med. 59 (4), 307-314.

Ndlovu, N., Rees, D., Murray, J., Vorajee, N., Richards, G., te WaterNaude, J., 2017. Asbestos-related diseases in mineworkers: a clinicopathological study. ERJ Open Res. 3(3).

Nelson, G., Girdler-Brown, B., Ndlovu, N., Murray, J., 2010. Three decades of silicosis: disease trends at autopsy in South African gold miners. Environ. Health Perspect. 118 (3), 421-426.

Nelson, G., Murray, J., Phillips, J.I., 2011. The risk of asbestos exposure in South African diamond mine workers. Ann. Occup. Hyg. 55 (6), 569-577.

Ouzzani, M., et al., 2016. Rayyan - a web and mobile app for systematic reviews. Syst. Rev. 5 (1), 210

Pachito, D.V., Bakusic, J., Boonen, E., et al., 2018. WHO/ILO work-related burden of disease and injury: Protocol for systematic reviews of exposure to long working hours and of the effect of exposure to long working hours on alcohol use and alcohol use disorder. Environ. Int (Submitted).

Pega, F., Blakely, T., Glymour, M.M., Carter, K.N., Kawachi, I., 2016. Using marginal structural modeling to estimate the cumulative impact of an unconditional tax credit on self-rated health. Am. J. Epidemiol. 183 (4), 315-324.

Pruss-Ustun, A., Wolf, J., Corvalan, C., Bos, R., Neira, M., 2017. In: Department of Public Health EaSDoH (Ed.), Preventing Disease Through Healthy Environments: A Global Assessment of the Burden of Disease From Environmental Risks. World Health Organization, Geneva.

Rehfuess, E.A., Booth, A., Brereton, L., et al., 2018. Towards a taxonomy of logic models in systematic reviews and health technology assessments: a priori, staged, and iterative approaches. Res. Synth. Methods 9 (1), 13-24.

Reiser, K., Hesterberg, T., Haschek, W., Last, J., 1982. Experimental silicosis. I. Acute effects of intratracheally instilled quartz on collagen metabolism and morphologic characteristics of rat lungs. Am. J. Pathol. 107 (2), 176.

Reiser, K., Haschek, W., Hesterberg, T., Last, J., 1983. Experimental silicosis. II. Longterm effects of intratracheally instilled quartz on collagen metabolism and morphologic characteristics of rat lungs. Am. J. Pathol. 110 (1), 30.

Review Manager (RevMan), 2014. Version 5.3. The Nordic Cochrane Centre, The Cochrane Collaboration, Copenhagen.

Rooney, A.A., Cooper, G.S., Jahnke, G.D., et al., 2016. How credible are the study results? Evaluating and applying internal validity tools to literature-based assessments of environmental health hazards. Environ. Int. 92-93, 617-629.

Rugulies, R.F., Ando, E., Ayuso Mateos, J.L., et al., 2018. WHO/ILO work-related burden of disease and injury: Protocol for systematic reviews of exposure to long working hours and of the effect of exposure to long working hours on depression. Environ. Int (Submitted).
Ryder, G., 2017. Welcome address from the Director General of the International Labour Organization. In: XXI World Congress on Safety and Health at Work; 2017. Sands Expo and Convention Centre, Singapore.

Schünemann, H., Oxman, A., Vist, G., et al., 2011. Chapter 12: interpreting results and drawing conclusions. In: Higgins, J., Green, S. (Eds.), Cochrane Handbook for Systematic Reviews of Interventions Version 510. The Cochrane Collaboration ([updated March 2011]. Available from www.handbook.cochrane.org).

Shamseer, L., Moher, D., Clarke, M., et al., 2015. Preferred reporting items for systematic review and meta-analysis protocols (PRISMA-P) 2015: elaboration and explanation. BMJ 349, g7647.

Shimizu, Y., Dobashi, K., Nagase, H., et al., 2015. Co-localization of iron binding on silica with p62/sequestosome1 (SQSTM1) in lung granulomas of mice with acute silicosis. J. Clin. Biochem. Nutr. 56 (1), 74-83.

Stata Cooperation, 2017. Stata 15. Version 6.0 ed. Stata Cooperation, College Station, TX.

Stevens, G.A., Alkema, L., Black, R.E., et al., 2016. Guidelines for accurate and transparent health estimates reporting: the GATHER stsstement. Lancet 388 (10062), e19-e23.

Sullivan, D.E., Ferris, M., Pociask, D., Brody, A.R., 2005. Tumor necrosis factor-alpha induces transforming growth factor-beta1 expression in lung fibroblasts through the extracellular signal-regulated kinase pathway. Am. J. Respir. Cell Mol. Biol. 32 (4), 342-349.

Tarres, J., Alberti, C., Martinez-Artes, X., et al., 2013. Pleural mesothelioma in relation to meteorological conditions and residential distance from an industrial source of asbestos. Occup. Environ. Med. 70 (8), 588-590.

Teixeira, L.R., Azevedo, T.M., Bortkiewicz, A.T., et al., 2018. WHO/ILO work-related burden of disease and injury: Protocol for systematic reviews of occupational exposure to noise and of the effect of occupational exposure to noise on cardiovascular disease. Environ. Int (Submitted).

Tenkate, T.D., Adam, B., Al Rifai, R.H., et al., 2018. WHO/ILO work-related burden of disease and injury: Protocol for systematic reviews of occupational exposure to solar ultraviolet radiation and of the effect of occupational exposure to solar ultraviolet radiation on cataract. Environ. Int (Submitted).

The GATHER Working Group, 2016. The GATHER Statement: Explanation and Elaboration. World Health Organization, Geneva.

United Nations, 2008. In: Affairs DoEaS (Ed.), ISIC Rev. 4: International Standard Industrial Classification of All Economic Activities, Revision 4. Statistical Papers Series M No. 4/Rev.4. New York, NY, United Nations.

Vandenberg, L.N., Agerstrand, M., Beronius, A., et al., 2016. A proposed framework for the systematic review and integrated assessment (SYRINA) of endocrine disrupting chemicals. Environ. Health 15 (1), 74.

Vesterinen, H., Johnson, P., Atchley, D., et al., 2014. The relationship between fetal growth and maternal glomerular filtration rate: a systematic review. J. Matern. Fetal Neonatal Med. 1-6.

Viswanathan, M., Ansari, M.T., Berkman, N.D., et al., 2008. Assessing the Risk of Bias of Individual Studies in Systematic Reviews of Health Care Interventions. Methods Guide for Effectiveness and Comparative Effectiveness Reviews. Rockville (MD).

Woodruff, T.J., Sutton, P., 2014. The navigation guide systematic review methodology: a rigorous and transparent method for translating environmental health science into better health outcomes. Environ. Health Perspect. 122 (10), 1007-1014.

World Health Organization, 2015. ICD-10: International Statistical Classification of Diseases and Related Health Problems: 10th Revision. World Health Organization, Geneva.

World Health Organization, 2017. In: Department of Information EaR (Ed.), WHO Methods and Data Sources for Global Burden of Disease Estimates 2000-2015. Global Health Estimates Technical Paper WHO/HIS/IER/GHE/2017.1. World Health Organization, Geneva. 